\title{
Comparative analysis of silage fermentation and in vitro digestibility of tropical grass prepared with Acremonium and Tricoderma species producing cellulases
}

\author{
Waroon Khota', Suradej Pholsen', David Higgs' ${ }^{2}$, and Yimin Cai ${ }^{3}$ **
}

\begin{abstract}
* Corresponding Author: Yimin Cai
Tel: +81-298386365, Fax: +81-298386653,

E-mail: cai@affrc.go.jp
\end{abstract}

' Faculty of Agriculture, Khon Kaen University, Khon Kaen 40002, Thailand

${ }^{2}$ Department of Biological and Environmental

Sciences, University of Hertfordshire, AL10 9AB, UK ${ }^{3}$ Japan International Research Center for Agricultural Science (JIRCAS), Tsukuba, Ibaraki 305-8686, Japan

ORCID

Waroon Khota

https://orcid.org/0000-0002-6766-9070 Suradej Pholsen

https://orcid.org/0000-0003-2746-7084 David Higgs

https://orcid.org/0000-0001-5534-1345

Yimin Cai

https://orcid.org/0000-0003-2650-5210

Submitted Jan 22, 2018; Revised Mar 22, 2018; Accepted May 11, 2018
Objective: To find out ways of improving fermentation quality of silage, the comparative analysis of fermentation characteristics and in vitro digestibility of tropical grasses silage applied with cellulases produced from Acremonium or Tricoderma species were studied in Thailand. Methods: Fresh and wilted Guinea grass and Napier grass silages were prepared with cellulases from Acremonium (AC) or Trichoderma (TC) at $0.0025 \%, 0.005 \%$, and $0.01 \%$ on a fresh matter (FM), and their fermentation quality, chemical composition and in vitro digestibility were analyzed.

Results: All silages of fresh Napier grass were good quality with lower $\mathrm{pH}$, butyric acid, and ammonia nitrogen, but higher lactic acid content than wilted Napier grass and Guinea grass silage. Silages treated with AC $0.01 \%$ had the best result in terms of fermentation quality. They also had higher in vitro dry matter digestibility and in vitro organic matter digestibility at 6 and $48 \mathrm{~h}$ after incubation than other silages. Silages treated with lower levels at $0.005 \%$ or $0.0025 \%$ of AC and all levels of TC did not improve silage fermentation.

Conclusion: The AC could improve silage fermentation and in vitro degradation of Guinea grass and Napier grass silages, and the suitable addition ration is $0.01 \%(73.5 \mathrm{U})$ of FM for tropical silage preparation.

Keywords: Cellulase; In vitro Digestibility; Silage Fermentation; Tropical Grass

\section{INTRODUCTION}

Purple Guinea grass (Panicum maximum cv. TD 58) and Napier grass (Pennisetum purpureum $\times$ Pennisetum americanum $\mathrm{cv}$. Pak Chong 1 ) are widespread throughout tropical and subtropical areas. They adapt and grow well in a variety of soil types, are tolerant to different conditions and require quite low inputs for growth [1-3]. Both grasses are important roughage sources for ruminant feed because of high dry matter biomass production; ranged from 15 to $18 \mathrm{t} /$ ha per year for Guinea grass [1] and 63 to $87 \mathrm{t} /$ ha per year for Napier grass [3,4]. However, both grasses are seasonal with high dry matter yield over the short rainy season; thus, silage making is an increasingly important method to preserve fresh forages for feeding ruminants year-round [5]. It is well known to be difficult to make good quality silage with these species because they are usually low in water soluble carbohydrate (WSC) content, insufficient for rapid decrease of silage $\mathrm{pH}$. They are also low in dry matter and high in fiber content which promotes the growth of clostridia and decreases nutrient utilization of ruminants [6-8].

Cellulase enzymes are popular biological products for improving the fermentation quality of silages. It is widely accepted that they can improve the fermentation process of both grasses and legumes and thus enhance cattle performance [6-8]. The two main reasons for cellulase 
enzyme addition in silage making are firstly, to enhance plant fiber degradation to increase the content of WSC as a lactic acid bacteria (LAB) substrate to produce lactic acid and enhance the fermentation process [9-11] and secondly, to break down the structural carbohydrate component thus improving silage digestibility $[10,12,13]$. Many microorganisms that produce various cellulolytic enzymes have been studied for several decades. The genus of Acremonium and Trichoderma has been especially famous for producing cellulolytic enzymes with relatively high enzymatic activity [14]. There is increasing interest in applying cellulase enzymes at ensiling due to reports that it can improve the fermentation quality of silage $[15,16]$.

However, there is limited information available for comparative analysis of silage prepared with these cellulases produced from Acremonium and Trichoderma. The objectives of this study were to determine the fermentation quality and in vitro digestibility of tropical grass silage prepared with two cellulases at different concentration.

\section{MATERIALS AND METHODS}

\section{Forage sample and silage preparation}

Purple Guinea grass (Panicum maximum cv. TD 58) and Napier (Pennisetum purpureum $\times$ Pennisetum americanum $\mathrm{cv}$. Pak Chong 1) grass were grown in a Korat soil series (Oxic Paleustults) at the experimental farm, Faculty of Agriculture, Khon Kaen University, Thailand in May 2014. Before planting, the plot for Guinea grass was ploughed twice and harrowed once. Root stock of purple Guinea grass was planted by hand with a spacing of $75 \times 75 \mathrm{~cm}$ in row and between rows in a $10 \times$ $30 \mathrm{~m}$ of the rectangular plot. The plot was fertilized with cattle manure at a rate of $24,000 \mathrm{~kg} / \mathrm{ha}$ which 4 separate equal portions of $6,000 \mathrm{~kg} / \mathrm{ha}$ split applied for 4 cuts [16,17]. For Napier grass, one week before planting, the plot was ploughed and fertilized with basal fertilizers of NPK (15-15-15) and cattle manure at 300 and 12,500 kg/ha, respectively. The 11,111 stem cuttings of Napier grass were planted by hand with a spacing of $120 \times 75 \mathrm{~cm}$ in row and between rows in a total area of 1,600 $\mathrm{m}^{2}$. Two weeks after planting, the plot was weeded out and nitrogen fertilizer (urea) applied at a rate of $60 \mathrm{~kg} / \mathrm{ha}$ [4]. On 20 April 2015, guinea grass was cut to adjust the height to 10 $\mathrm{cm}$ above ground level and Napier grass was cut close to the soil surface. After $60 \mathrm{~d}$ of regrowth, both grasses were harvested in early morning and immediately chopped using a forage chopper (Supachai, Kanchanaburi, Thailand) to $1 \mathrm{~cm}$ particle size. Half of each chopped grass sample was wilted for $6 \mathrm{~h}$ in the shade to study the effect of moisture adjustment. Both fresh or wilted forages were then treated as follows: control (untreated), Acremonium cellulase (AC, Meiji Seika Pharma Co., Ltd, Tokyo, Japan): AC 0.0025\%, AC 0.005\%, AC 0.01\%, Trichoderma cellulase (TC, Meiji Seika Pharma Co., Ltd, Japan): TC $0.0025 \%$, TC $0.005 \%$, and TC $0.01 \%$. The pro- duction strain, main composition and carboxymethyl-cellulase (CMCase) of AC were Acremonium cellulolyticus, glucanase and pectinase, and 7,350 U/g; for TC they were Tricoderma viride, xylanase and glucanase, and 2,720 U/g, respectively. Treatments were ensiled using a small scale fermentation system [18]. Both AC and TC cellulases were added as $\%$ of fresh matter (FM). The design of this experiment was a 2 (two grasses) $\times 2$ (fresh vs wilted) $\times 2$ (two enzymes) $\times 3$ (three application levels) factorial arrangement in a completely randomized design with triplicates for each treatment. The silage was prepared with a laboratory-scale fermentation method [16]. The mixed grass with additive (1,000 g) was packed into a synthetic silo laminated from nylon and polyethylene (Hiryu $\mathrm{KN}$ type, Asahikasei, Tokyo, Japan), and sealed by using a vacuum sealer (SQ-303, Asahi Kasei Pax Corp., Japan). All silos were stored at room temperature $\left(25^{\circ} \mathrm{C}\right.$ to $\left.37^{\circ} \mathrm{C}\right)$. On $30 \mathrm{~d}$ of ensiling, silos were opened for evaluation of fermentation products, chemical composition, and in vitro digestibility.

\section{Microorganism analysis}

Microorganism numbers were analyzed using the plate count method as described by [19], and reported as colony-forming unit (cfu)/g of FM. Fresh or wilted forage samples $(1,000 \mathrm{~g})$ or silage samples (200 g) were sub-sampled for $10 \mathrm{~g}$ samples. Then, each sample (10 g) was homogenized with $90 \mathrm{~mL}$ sterilized distilled water, and serial dilutions in $0.85 \%$ sodium chloride solution at $10^{-1}$ to $10^{-5}$. Each dilution $(20 \mu \mathrm{L})$ was dropped and spread on prepared agar plates. MRS agar (Difco Lab $\neg$ oratories, Detroit, MI, USA) was used for the LAB count, which the agar plates were put into an anaerobic box (TE-HER Hard Anaerobox, ANX-1; Hirosawa Ltd., Tokyo, Japan), and incubation at $30^{\circ} \mathrm{C}$ for $48 \mathrm{~h}$ in an incubator. Blue light broth agar (Nissui-seiyaku Ltd., Tokyo, Japan) was used for coliform bacteria counts after incubating at $30^{\circ} \mathrm{C}$ for $48 \mathrm{~h}$. Nutrient agar (Difco, USA) and potato dextrose agar (Nissui-seiyaku, Japan) were used for aerobic bacteria, yeast, and mold. Mold was counted after $2 \mathrm{~d}$ of incubation. Yeasts were distinguished from molds or bacteria by colony appearance and cell morphology observation.

\section{Chemical composition}

Cold water extraction of silage samples was used for fermentation end-product analysis following the method of Cai [20]. Ten grams of wet silage was soaked with $90 \mathrm{~mL}$ sterilized distilled water, and then, incubated at $4^{\circ} \mathrm{C}$ in a refrigerator overnight. Silage $\mathrm{pH}$ was measured immediately after incubating using a glass electrode $\mathrm{pH}$ meter (FiveGo; Mettler Toledo, Greifensee, Switzerland), and $10 \mathrm{~mL}$ of cold water extract was sampled and stored at $-20^{\circ} \mathrm{C}$ for further analysis of organic acids and ammonia nitrogen concentrations. Organic acid content of silages was measured by HPLC methods [20]. Ammonia nitrogen concentration was analyzed using 
a spectrophotometer (UV/VIS Spectrometer, PG Instruments Ltd., London, UK) [21]. The lactate buffering capacity (LBC) of forages was analyzed by the titration method viz. $10 \mathrm{~g}$ of sample homogenized with distilled water, titrated with $0.1 \mathrm{M}$ hydrochloric acid $(\mathrm{HCl})$ to decrease $\mathrm{pH}$ from initial $\mathrm{pH}$ to 3 , then titrated to $\mathrm{pH} 6$ with $0.1 \mathrm{M}$ sodium hydroxide $(\mathrm{NaOH})$ [13]. The WSC content of forage was extracted and measured following the methods of [16].

Forage and silage samples on $30 \mathrm{~d}$ of ensiling were dried at $60^{\circ} \mathrm{C}$ for $48 \mathrm{~h}$ in a hot air oven, and ground through a $1 \mathrm{~mm}$ mesh screen. Dry matter (DM), organic matter (OM), crude protein $(\mathrm{CP})$, and ether extract (EE) were analyzed following methods 934.01, 942.05, 976.05, and 920.39, respectively [22]. Neutral detergent fiber (NDF), acid detergent fiber (ADF), and acid detergent lignin (ADL) were analyzed using a fiber analyzer (ANKOM 200, ANKOM Technology, New York, USA), based on the method of [23].

\section{Animal care}

The animal experiment was approved by the Animal Care and Use Committee of Khon Kaen University (KKU), Khon Kaen, Thailand and performed at KKU in August 2015. The experiment was performed according to recommendations proposed by the European Commission [24] and to minimize the suffering of animals.

\section{In vitro digestibility analysis}

In vitro digestibility of DM (IVDMD) and OM (IVOMD) were measured after incubating samples in buffered rumen fluid at 6 and $48 \mathrm{~h}$. The buffered rumen inoculum was prepared following the method of [25]. Rumen fluid was collected from two dairy steers by a stomach tube sucker before morning feeding. The $\mathrm{pH}$ of rumen fluid was 7.2. Ground silage samples $(0.5 \mathrm{~g})$ was put into serum bottles of $50 \mathrm{~mL}$ capacity ( 3 replications per sample). The bottles were closed by a rubber stopper with an aluminum seal cap. The buffered rumen inoculum $(40 \mathrm{~mL})$ was injected into each sample bottle using a $60 \mathrm{~mL}$ syringe (Nipro Thailand corporation, Ltd., Phra Nakhon Si Ayutthaya, Thailand) with 18 gauge $\times 3.5 \mathrm{~cm}$ needle (Nipro Corporation, Osaka, Japan), and flushed with carbon dioxide gas to produce an anaerobic condition. All samples were incubated in a water bath at $39^{\circ} \mathrm{C}$, swirled by hand and the head space gas production released using a 21 gauge $\times 3.5 \mathrm{~cm}$ needle (Nipro, Japan) at $2 \mathrm{~h}$ intervals. Two blanks of $40 \mathrm{~mL}$ of rumen inoculum per bottle were also incubated.

The animals were housed in individual pen equipped with mineral blocks (FNZ Red Lick, Thai Serve Co. Ltd., Buriram, Thailand; mineral composition: $\mathrm{NaCl} 930.00$ g, $\mathrm{Mg} 2.00$ g, Zn 0.77 g, Mn 0.50 g, Co 18.00 g, I 0.05 g, Se 0.01 g, Cu 0.22 g, another $2.50 \mathrm{~g})$ and fresh water, and fed concentrate $(16 \% \mathrm{CP}$, $11.0 \mathrm{MJ} / \mathrm{kg}$ of metabolizable energy, feed ingredient including: $10 \%$ palm kernel meal, $7 \%$ coconut meal, $6 \%$ rice bran,
$70 \%$ cassava chip, $4 \%$ urea, $1 \%$ sulfur, $1 \%$ mineral premix, and $1 \%$ salt on a DM basis) at $0.5 \%$ of body weight and ad libitum rice straw daily. The diet was fed in two equal meals at 08:00 and 16:00 h.

\section{Statistical analysis}

Data on fermentation products, chemical composition, and in vitro digestibility of silages at $30 \mathrm{~d}$ after fermentation were analyzed using a completely randomized design with a $2 \times 2 \times$ $2 \times 3$ (forages $[\mathrm{A}] \times$ moisture adjustment $[\mathrm{B}] \times$ enzymes $[\mathrm{C}] \times$ application levels [D]) factorial treatment combinations. The analysis of variance procedure of SAS version 6.12 (SAS Institute Inc., Cary, NC, USA) was used for the analysis and the statistical model is as follows:

$$
\mathrm{Y}_{\mathrm{ijklm}}=\mu+\alpha_{\mathrm{i}}+\beta_{\mathrm{j}}+\gamma_{\mathrm{k}}+\delta_{\mathrm{l}}+\alpha \beta \gamma \delta_{\mathrm{ijkl}}+\varepsilon_{\mathrm{ijklm}}
$$

Where $\mathrm{Y}_{\mathrm{i} \mathrm{jk} m}=$ observation; $\mu$ = overall mean, $\alpha_{\mathrm{i}}=$ forage effect ( $\mathrm{i}=$ Guinea grass and Napier grass), $\beta_{\mathrm{j}}=$ moisture adjustment effect $(\mathrm{j}=$ fresh and wilted $), \gamma_{\mathrm{k}}=$ enzyme effect (AC and TC), $\delta_{1}=$ application levels $(0.0025 \%, 0.005 \%$, and $0.01 \%)$, $\alpha \beta \gamma \delta_{\mathrm{ijkl}}=$ forages $\times$ moisture adjustment $\times$ enzymes $\times$ application levels, and $\varepsilon_{\mathrm{ijklm}}=$ error. The significant difference among treatment means was tested by Duncan's new multiple range test at $\mathrm{p}=0.05[26]$.

\section{RESULTS}

\section{Microorganism population and chemical composition of forages}

The microorganism counts of forages are shown in Table 1. Fresh Guinea grass and fresh Napier grass showed similar numbers of LAB $\left(10^{3} \mathrm{cfu} / \mathrm{g}\right.$ of FM), aerobic bacteria $\left(10^{7} \mathrm{cfu} / \mathrm{g}\right.$ of FM), and coliform bacteria ( $10^{7} \mathrm{cfu} / \mathrm{g}$ of FM). The counts of yeasts and molds of both fresh grasses ranged from $10^{4}$ to $10^{5}$ and $10^{2}$ to $10^{3} \mathrm{cfu} / \mathrm{g}$ of $\mathrm{FM}$, respectively. After wilting process, the counts of $\mathrm{LAB}$, yeasts and molds of both grasses increased from $10^{4}$ to $10^{5}, 10^{5}$ to $10^{6}$, and $10^{4} \mathrm{cfu} / \mathrm{g}$ of $\mathrm{FM}$, respectively, the counts of coliform bacteria and aerobic bacteria did not change by the wilting process.

Table 1. Microorganism counts (cfu/g of FM) of Guinea grass and Napier grass before ensiling

\begin{tabular}{lccccc}
\hline Items & $\begin{array}{c}\text { Lactic acid } \\
\text { bacteria }\end{array}$ & $\begin{array}{c}\text { Coliform } \\
\text { bacteria }\end{array}$ & $\begin{array}{c}\text { Aerobic } \\
\text { bacteria }\end{array}$ & Yeast & Mold \\
\hline $\begin{array}{l}\text { Guinea grass } \\
\quad \text { Fresh }\end{array}$ & $1.8 \times 10^{3}$ & $1.5 \times 10^{7}$ & $2.6 \times 10^{7}$ & $2.9 \times 10^{4}$ & $1.3 \times 10^{3}$ \\
$\quad$ Wilted & $1.2 \times 10^{4}$ & $4.2 \times 10^{7}$ & $2.4 \times 10^{7}$ & $1.5 \times 10^{5}$ & $1.6 \times 10^{4}$ \\
Napier grass & & & & & \\
$\quad$ Fresh & $2.5 \times 10^{3}$ & $8.1 \times 10^{7}$ & $1.1 \times 10^{7}$ & $1.5 \times 10^{5}$ & $2.5 \times 10^{2}$ \\
$\quad$ Wilted & $3.5 \times 10^{5}$ & $6.2 \times 10^{7}$ & $2.0 \times 10^{7}$ & $2.0 \times 10^{6}$ & $2.9 \times 10^{4}$ \\
\hline cfu, colony-forming unit; FM, fresh matter. & & &
\end{tabular}


The chemical composition and LBC of Guinea grass and Napier grass are shown in Table 2. The OM content was higher $(\mathrm{p}<0.01)$ and WSC content was lower $(\mathrm{p}<0.01)$ in Napier grasses than those in Guinea grasses. During the wilting process, the DM of Guinea grass and Napier grass significantly $(\mathrm{p}<0.01)$ increased by $7 \%$ and $8 \%$, respectively, but the contents of $\mathrm{ADL}$ and LBC contents did not differ. Compared with fresh Guinea grass, the contents of $\mathrm{CP}$ and $\mathrm{ADF}$ were higher $(\mathrm{p}<0.01)$, but the NDF content was lower in wilting Guinea grass. Also, wilted Napier grass showed higher $(\mathrm{p}<0.01)$ CP content and lower EE content than fresh Napier grass.

\section{Fermentation quality of silages}

The $\mathrm{DM}, \mathrm{pH}$, and fermentation products of Guinea grass and Napier grass silages at $30 \mathrm{~d}$ of ensiling are shown in Table 3. The interaction between $\mathrm{A}, \mathrm{B}, \mathrm{C}$, and $\mathrm{D}$ influenced $(\mathrm{p}<0.01)$ the silage $\mathrm{DM}, \mathrm{pH}$, all organic acid contents, and ammonia nitrogen content. Guinea grass silage treated with AC 0.01\% showed good quality with lower $(\mathrm{p}<0.01) \mathrm{pH}$ and ammonia nitrogen, and higher $(\mathrm{p}<0.01)$ lactic acid content than other silages. Regarding the differences in production strain $(A$. cellulolyticus vs T. viride), main composition (glucanase and pectinase, vs xylanase and glucanase), and CMCase (7,350 vs $2,720 \mathrm{U} / \mathrm{g})$ between $\mathrm{AC}$ and $\mathrm{TC}$, the highest $(\mathrm{p}<0.01) \mathrm{pH}$ and ammonia nitrogen content with lowest $(\mathrm{p}<0.01)$ lactic acid content were found in wilted Guinea grass silages treated with TC $0.05 \%$. The AC treatments improved grass silage quality more than TC treatments. The addition level of AC at $0.01 \%$ improved fermentation quality more than $0.005 \%$ and $0.0025 \%$.

\section{Microorganism counts of silages}

The microbiological analysis of Guinea grass and Napier grass silages at $30 \mathrm{~d}$ of ensiling are shown in Table 4 . The A, B, C, D and their interaction $(A \times B \times C \times D)$ were not significantly different $(\mathrm{p}<0.05)$ in $\mathrm{LAB}$ count, aerobic bacteria and yeasts. Coliform bacteria and molds were below the detectable level $\left(10^{2} \mathrm{cfu} / \mathrm{g}\right.$ of FM) in all silages. The LAB population of all silages was dominant ranging from $10^{7}$ to $10^{8} \mathrm{cfu} / \mathrm{g}$ of FM. Aerobic bacteria were $10^{3}$ to $10^{4} \mathrm{cfu} / \mathrm{g}$ of FM and yeast were $10^{2}$ to $10^{4}$ cfu/g of FM.

\section{Chemical composition of silages}

The chemical compositions of Guinea grass and Napier grass silages at $30 \mathrm{~d}$ of ensiling are shown in Table 5 . The significances of $\mathrm{A} \times \mathrm{B} \times \mathrm{C} \times \mathrm{D}(\mathrm{p}<0.001)$ influenced all chemical compositions. The OM content of AC or TC treated Guinea grass silage were higher $(p<0.01)$ than other silages. The wilted Napier grass silages treated with $\mathrm{AC} 0.01 \%$ showed higher $(\mathrm{p}<0.01)$ CP content and lower $(\mathrm{p}<0.01) \mathrm{NDF}$ and ADF content than other silages. The highest $(\mathrm{p}<0.01)$ fiber contents were observed in TC treated fresh Guinea grass silages. When silages treated with $\mathrm{AC}$, the $\mathrm{CP}, \mathrm{EE}$, and $\mathrm{ADL}$ contents were significantly ( $\mathrm{p}<$ 0.01 ) higher and the NDF and ADF contents were significantly $(\mathrm{p}<0.01)$ lower than TC treatments; and the application levels of enzymes at $0.01 \%$ decreased fiber content more than $0.005 \%$ and $0.0025 \%$.

\section{In vitro digestibility of silages}

The in vitro digestibility of Guinea grass and Napier grass silages at $30 \mathrm{~d}$ of ensiling are shown in Table 6. Fresh Napier grass silages treated with AC $0.01 \%$ had higher $(\mathrm{p}<0.05)$ IVDMD than other treatments. Napier grass silages had higher $(\mathrm{p}<0.01)$ IVDMD and IVOMD than Guinea grass silages. The wilted grasses silages had higher $(\mathrm{p}<0.05)$ IVOMD than fresh grasses silages. When silages treated with $\mathrm{AC}$, the IVDMD and IVOMD at $6 \mathrm{~h}$ after incubation were significantly higher $(\mathrm{p}<0.01)$ than those of TC treatments. The IVDMD and IVOMD of $0.01 \%$ cellulase treated silage at 6 and $48 \mathrm{~h}$ after incubation were greater $(\mathrm{p}<0.05)$ than those of $0.0025 \%$. The significances of $\mathrm{A} \times \mathrm{B} \times \mathrm{C} \times \mathrm{D}(\mathrm{p}<0.05)$ influenced IVDMD at $48 \mathrm{~h}$ after incubation in buffered rumen fluid, while IVDMD at $6 \mathrm{~h}$ after incubation and IVOMD at both 6 and $48 \mathrm{~h}$ after incubation did not.

Table 2. Chemical composition (\% of DM) and lactate buffer capacity (LBC, $\mathrm{mE} / \mathrm{kg}$ of DM) of Guinea grass and Napier grass before ensiling

\begin{tabular}{lccccccccc}
\hline Items & DM & OM & CP & EE & NDF & ADF & ADL & WSC & LBC \\
\hline Guinea grass & & & & & & & & & \\
$\quad$ Fresh & $17.81^{\mathrm{c}}$ & $90.16^{\mathrm{a}}$ & $7.49^{\mathrm{c}}$ & $1.65^{\mathrm{b}}$ & $77.47^{\mathrm{a}}$ & $48.26^{\mathrm{b}}$ & 3.32 & $0.48^{\mathrm{b}}$ & 760.81 \\
$\quad$ Wilted & $24.78^{\mathrm{a}}$ & $89.76^{\mathrm{a}}$ & $8.21^{\mathrm{b}}$ & $1.63^{\mathrm{b}}$ & $76.24^{\mathrm{b}}$ & $51.45^{\mathrm{a}}$ & 2.76 & $0.39^{\mathrm{b}}$ & 526.64 \\
$\quad$ Napier grass & & & & & & & & & \\
$\quad$ Fresh & $13.08^{\mathrm{d}}$ & $87.23^{\mathrm{b}}$ & $7.19^{\mathrm{c}}$ & $2.07^{\mathrm{a}}$ & $71.14^{\mathrm{c}}$ & $42.81^{\mathrm{c}}$ & 3.18 & $2.59^{\mathrm{a}}$ & 722.48 \\
$\quad$ Wilted & $21.86^{\mathrm{b}}$ & $87.62^{\mathrm{b}}$ & $8.70^{\mathrm{a}}$ & $1.66^{\mathrm{b}}$ & $70.82^{\mathrm{c}}$ & $39.11^{\mathrm{d}}$ & 2.92 & $2.46^{\mathrm{a}}$ & 622.15 \\
SEM & 1.020 & 0.154 & 0.119 & 0.091 & 0.237 & 0.452 & 3.32 & 0.058 & 75.167 \\
p-value & 0.001 & $<0.001$ & $<0.001$ & 0.022 & $<0.001$ & $<0.001$ & 0.411 & $<0.001$ & 0.110 \\
\hline
\end{tabular}

DM, dry matter; OM, organic matter; CP, crude protein; EE, ether extract; NDF, neutral detergent fiber; ADF, acid detergent fiber; ADL, acid detergent lignin; WSC, water soluble carbohydrate; SEM, standard error of the mean.

a-d Means within columns with different superscripts differ at $p<0.05$. 
Table 3. Dry matter (\%), pH and fermentation products ( $\mathrm{g} / \mathrm{kg}$ of $\mathrm{DM}$ ) of Guinea grass and Napier grass silages at $30 \mathrm{~d}$ after fermentation

\begin{tabular}{|c|c|c|c|c|c|c|c|}
\hline Treatments & $\mathrm{DM}$ & $\mathrm{pH}$ & Lactic acid & Acetic acid & Propionic acid & Butyric acid & Ammonia nitrogen \\
\hline \multicolumn{8}{|l|}{ Fresh Guinea } \\
\hline AC $0.0025 \%$ & $18.43^{c}$ & $4.56^{b c}$ & $0.10^{\mathrm{h}}$ & $4.42^{b}$ & $0.21^{b}$ & $3.09^{\mathrm{a}}$ & $0.99^{\text {eggh }}$ \\
\hline AC $0.005 \%$ & $17.09^{\text {cd }}$ & $4.49^{c}$ & $0.52^{\text {gh }}$ & $3.92^{c}$ & $0.13^{c}$ & $3.28^{\mathrm{a}}$ & $0.88^{\text {fghi }}$ \\
\hline AC $0.01 \%$ & $18.34^{c}$ & $3.55^{i}$ & $6.71^{\mathrm{ab}}$ & $1.79^{\mathrm{ef}}$ & $0.02^{\mathrm{efg}^{\mathrm{f}}}$ & $0.18^{\text {defg }}$ & $0.18^{\mathrm{j}}$ \\
\hline TC $0.0025 \%$ & $17.09^{\mathrm{cd}}$ & $4.59^{b c}$ & $0.00^{\mathrm{h}}$ & $5.27^{\mathrm{a}}$ & $0.32^{\mathrm{a}}$ & $2.66^{\mathrm{b}}$ & $1.49^{\text {bcde }}$ \\
\hline TC $0.005 \%$ & $18.18^{c}$ & $4.63^{\mathrm{bc}}$ & $0.01^{\mathrm{h}}$ & $4.71^{b}$ & $0.35^{\mathrm{a}}$ & $2.23^{c}$ & $1.20^{\text {cdef }}$ \\
\hline TC $0.01 \%$ & $17.60^{c}$ & $4.60^{b c}$ & $0.00^{h}$ & $5.18^{\mathrm{a}}$ & $0.21^{b}$ & $2.62^{b}$ & $1.63^{\text {bcd }}$ \\
\hline \multicolumn{8}{|l|}{ Wilted Guinea } \\
\hline AC $0.0025 \%$ & $23.84^{\mathrm{a}}$ & $4.66^{b c}$ & $1.33^{\text {efgh }}$ & $1.15^{\mathrm{hi}}$ & $0.03^{\mathrm{efg}^{\mathrm{fg}}}$ & $0.18^{\text {defg }}$ & $1.78^{\mathrm{bc}}$ \\
\hline AC $0.005 \%$ & $23.46^{\mathrm{a}}$ & $4.49^{c}$ & $2.78^{\text {def }}$ & $1.74^{f g}$ & $0.03^{\mathrm{efg}^{\mathrm{fg}}}$ & $0.42^{\mathrm{d}}$ & $1.56^{\text {bcde }}$ \\
\hline AC $0.01 \%$ & $23.48^{\mathrm{a}}$ & $4.25^{\mathrm{de}}$ & $2.07^{\text {egh }}$ & $0.88^{i}$ & $0.02^{\mathrm{efg}^{\mathrm{f}}}$ & $0.06^{e^{f g}}$ & $1.69^{\mathrm{bc}}$ \\
\hline TC $0.0025 \%$ & $24.07^{\mathrm{a}}$ & $4.90^{\mathrm{a}}$ & $1.75^{\text {eggh }}$ & $2.31^{d}$ & $0.06^{\mathrm{d}}$ & $0.24^{\text {defg }}$ & $2.08^{b}$ \\
\hline TC $0.005 \%$ & $23.29^{\mathrm{a}}$ & $5.05^{\mathrm{a}}$ & $0.47^{\text {gh }}$ & $1.29^{\text {fghi }}$ & $0.05^{\mathrm{de}}$ & $0.42^{d}$ & $2.74^{\mathrm{a}}$ \\
\hline TC $0.01 \%$ & $23.65^{\mathrm{a}}$ & $4.91^{\mathrm{a}}$ & $0.71^{\text {foh }}$ & $1.18^{\mathrm{hi}}$ & $0.04^{\text {def }}$ & $0.21^{\text {defg }}$ & $1.74^{\mathrm{bc}}$ \\
\hline \multicolumn{8}{|l|}{ Fresh Napier } \\
\hline AC $0.0025 \%$ & $13.51^{\mathrm{ef}}$ & $3.89^{g^{h}}$ & $2.69^{\text {defig }}$ & $1.55^{\mathrm{fgh}}$ & $0.02^{\mathrm{efg}^{\mathrm{f}}}$ & $0.01^{9}$ & $0.42^{\text {ghij }}$ \\
\hline AC $0.005 \%$ & $12.64^{f}$ & $3.78^{\mathrm{h}}$ & $7.56^{\mathrm{a}}$ & $1.35^{\text {fohi }}$ & $0.00^{9}$ & $0.03^{f g}$ & $0.32^{\mathrm{j}}$ \\
\hline AC $0.01 \%$ & $15.06^{\mathrm{de}}$ & $3.87^{g^{\mathrm{h}}}$ & $5.52^{\mathrm{ab}}$ & $1.21^{9^{\mathrm{hi}}}$ & $0.01^{\mathrm{fg}}$ & $0.03^{\text {fg }}$ & $0.27^{j}$ \\
\hline TC $0.0025 \%$ & $18.08^{c}$ & $4.23^{\mathrm{de}}$ & $4.73^{\text {bcd }}$ & $1.68^{\text {ghh }}$ & $0.01^{\text {fg }}$ & $0.04^{f g}$ & $0.76^{\text {fohij }}$ \\
\hline TC $0.005 \%$ & $14.18^{\mathrm{ef}}$ & $4.08^{e f}$ & $5.51^{\mathrm{ab}}$ & $1.47^{\text {tgh }}$ & $0.01^{\mathrm{fg}}$ & $0.04^{f g}$ & $0.65^{\text {fohij }}$ \\
\hline TC $0.01 \%$ & $13.79^{\text {ef }}$ & $4.03^{\text {tg }}$ & $5.36^{\mathrm{bc}}$ & $1.22^{\text {ghi }}$ & $0.00^{9}$ & $0.02^{9}$ & $0.40^{\mathrm{hij}}$ \\
\hline \multicolumn{8}{|l|}{ Wilted Napier } \\
\hline AC $0.0025 \%$ & $23.46^{\mathrm{a}}$ & $4.33^{d}$ & $0.76^{\text {foh }}$ & $1.57^{\text {gh }}$ & $0.01^{\text {fg }}$ & $0.01^{9}$ & $0.74^{\text {fghij }}$ \\
\hline AC $0.005 \%$ & $22.52^{\mathrm{ab}}$ & $4.17^{\text {def }}$ & $4.85^{\text {bcd }}$ & $1.48^{\text {fgh }}$ & $0.01^{\mathrm{fg}}$ & $0.01^{9}$ & $0.67^{\text {fohij }}$ \\
\hline AC $0.01 \%$ & $23.58^{\mathrm{a}}$ & $4.09^{e f}$ & $5.07^{\mathrm{bc}}$ & $1.42^{\text {fgh }}$ & $0.00^{9}$ & $0.01^{9}$ & $0.77^{\text {fohij }}$ \\
\hline TC $0.0025 \%$ & $22.08^{\mathrm{ab}}$ & $4.68^{b}$ & $3.27^{\text {de }}$ & $2.48^{d}$ & $0.02^{e f g}$ & $0.35^{\mathrm{d}}$ & $1.03^{\mathrm{efg}^{\mathrm{g}}}$ \\
\hline TC $0.005 \%$ & $23.61^{\mathrm{a}}$ & $4.64^{b c}$ & $3.26^{\text {de }}$ & $2.23^{\mathrm{de}}$ & $0.01^{\text {fg }}$ & $0.08^{\text {efg }}$ & $1.04^{\mathrm{ef}}$ \\
\hline TC $0.01 \%$ & $21.06^{b}$ & $4.68^{\mathrm{b}}$ & $3.19^{\text {de }}$ & $2.51^{d}$ & $0.00^{9}$ & $0.33^{\text {def }}$ & $1.07^{\text {def }}$ \\
\hline SEM & 0.826 & 0.062 & 0.781 & 0.188 & 0.012 & 0.105 & 0.210 \\
\hline \multicolumn{8}{|l|}{ Grasses means } \\
\hline Guinea grass & $20.71^{\mathrm{a}}$ & $4.56^{\mathrm{a}}$ & $1.37^{b}$ & $2.82^{\mathrm{a}}$ & $0.12^{\mathrm{a}}$ & $1.30^{\mathrm{a}}$ & $1.50^{\mathrm{a}}$ \\
\hline Napier grass & $18.63^{b}$ & $4.21^{b}$ & $4.31^{\mathrm{a}}$ & $1.68^{\mathrm{b}}$ & $0.01^{b}$ & $0.08^{b}$ & $0.68^{b}$ \\
\hline \multicolumn{8}{|l|}{ Moisture adjust } \\
\hline Fresh & $16.17^{b}$ & $4.19^{b}$ & $3.23^{\mathrm{a}}$ & $2.82^{\mathrm{a}}$ & $0.11^{\mathrm{a}}$ & $1.19^{\mathrm{a}}$ & $0.77^{\mathrm{b}}$ \\
\hline Wilted & $23.18^{\mathrm{a}}$ & $4.57^{\mathrm{a}}$ & $2.46^{b}$ & $1.69^{b}$ & $0.02^{b}$ & $0.19^{b}$ & $1.41^{\mathrm{a}}$ \\
\hline \multicolumn{8}{|l|}{ Enzymes } \\
\hline$A C$ & 19.62 & $4.18^{b}$ & $3.33^{\mathrm{a}}$ & $1.87^{\mathrm{b}}$ & $0.04^{b}$ & $0.61^{b}$ & $0.86^{\mathrm{b}}$ \\
\hline $\mathrm{TC}$ & 19.73 & $4.58^{\mathrm{a}}$ & $2.35^{b}$ & $2.63^{\mathrm{a}}$ & $0.09^{a}$ & $0.77^{\mathrm{a}}$ & $1.32^{\mathrm{a}}$ \\
\hline \multicolumn{8}{|l|}{ Application levels } \\
\hline $0.0025 \%$ & 20.07 & $4.48^{\mathrm{a}}$ & $1.83^{\mathrm{c}}$ & $2.56^{\mathrm{a}}$ & $0.09^{\mathrm{a}}$ & $0.82^{\mathrm{a}}$ & 1.16 \\
\hline $0.005 \%$ & 19.37 & $4.42^{b}$ & $3.12^{b}$ & $2.27^{b}$ & $0.07^{b}$ & $0.81^{a}$ & 1.13 \\
\hline $0.01 \%$ & 19.57 & $4.25^{c}$ & $3.58^{\mathrm{a}}$ & $1.92^{\mathrm{c}}$ & $0.04^{c}$ & $0.43^{b}$ & 0.97 \\
\hline \multicolumn{8}{|c|}{ Significance of main effect and interaction } \\
\hline Grasses means (A) & $<0.001$ & $<0.001$ & $<0.001$ & $<0.001$ & $<0.001$ & $<0.001$ & $<0.001$ \\
\hline Moisture adjust (B) & $<0.001$ & $<0.002$ & 0.008 & 0.008 & $<0.001$ & $<0.001$ & $<0.001$ \\
\hline Enzymes (C) & 0.712 & $<0.003$ & 0.001 & 0.001 & $<0.001$ & $<0.001$ & $<0.001$ \\
\hline Application levels (D) & 0.141 & $<0.004$ & $<0.001$ & $<0.001$ & $<0.001$ & $<0.001$ & 0.087 \\
\hline$A \times B \times C \times D$ & 0.001 & $<0.005$ & $<0.001$ & $<0.001$ & $<0.001$ & $<0.001$ & 0.007 \\
\hline
\end{tabular}

DM, dry matter; AC, Acremonium cellulase (Meiji Seika Pharma Co. Ltd., Tokyo, Japan); TC, Trichoderma cellulase (Meiji Seika Pharma Co. Ltd., Japan); SEM, standard error of the mean.

${ }^{a-j}$ Means within columns with different superscripts differ at $p<0.05$.

Values are means of three silage samples.

\section{DISCUSSION}

Generally, the DM content of forages influences fermentation quality of the silage; and optimal DM content ranges from $30 \%$ to $40 \%$ for good quality silage making $[13,27]$. If dry matter content is less than $20 \%$, the fermentation process would be 
Table 4. Microbiological analysis of Guinea grass and Napier grass silages at 30 d after fermentation

\begin{tabular}{|c|c|c|c|c|c|}
\hline \multirow[b]{2}{*}{ Treatments } & \multicolumn{5}{|c|}{ Microorganism (cfu/g of FM) } \\
\hline & $\begin{array}{l}\text { Lactic acid } \\
\text { bacteria }\end{array}$ & $\begin{array}{l}\text { Coliform } \\
\text { bacteria }\end{array}$ & $\begin{array}{l}\text { Aerobic } \\
\text { bacteria }\end{array}$ & Yeast & Mold \\
\hline \multicolumn{6}{|l|}{ Fresh Guinea } \\
\hline AC $0.0025 \%$ & $9.0 \times 10^{7}$ & ND & $1.4 \times 10^{4}$ & ND & ND \\
\hline AC $0.005 \%$ & $3.9 \times 10^{8}$ & ND & $1.2 \times 10^{4}$ & ND & ND \\
\hline AC $0.01 \%$ & $2.8 \times 10^{8}$ & ND & $1.5 \times 10^{4}$ & ND & ND \\
\hline TC $0.0025 \%$ & $1.2 \times 10^{8}$ & ND & $3.5 \times 10^{3}$ & $1.9 \times 10^{2}$ & ND \\
\hline TC $0.005 \%$ & $7.9 \times 10^{7}$ & ND & $0.9 \times 10^{4}$ & $1.7 \times 10^{2}$ & ND \\
\hline TC $0.01 \%$ & $2.6 \times 10^{8}$ & ND & $1.9 \times 10^{4}$ & $2.3 \times 10^{2}$ & ND \\
\hline \multicolumn{6}{|l|}{ Wilted Guinea } \\
\hline AC $0.0025 \%$ & $2.4 \times 10^{8}$ & ND & $8.1 \times 10^{3}$ & ND & ND \\
\hline AC $0.005 \%$ & $7 \times 10^{7}$ & ND & $1.6 \times 10^{4}$ & ND & ND \\
\hline AC $0.01 \%$ & $1.5 \times 10^{8}$ & ND & $8.0 \times 10^{3}$ & ND & ND \\
\hline TC $0.0025 \%$ & $1.2 \times 10^{8}$ & ND & $6.8 \times 10^{3}$ & $4.6 \times 10^{2}$ & ND \\
\hline TC $0.005 \%$ & $3.7 \times 10^{8}$ & ND & $1.8 \times 10^{4}$ & $5.6 \times 10^{2}$ & $N D$ \\
\hline TC $0.01 \%$ & $1.7 \times 10^{8}$ & ND & $1.6 \times 10^{4}$ & $1.9 \times 10^{2}$ & ND \\
\hline \multicolumn{6}{|l|}{ Fresh Napier } \\
\hline AC $0.0025 \%$ & $3.0 \times 10^{7}$ & ND & $1.8 \times 10^{4}$ & ND & ND \\
\hline AC $0.005 \%$ & $1.6 \times 10^{8}$ & ND & $3.4 \times 10^{4}$ & $7.2 \times 10^{2}$ & ND \\
\hline AC $0.01 \%$ & $1.5 \times 10^{8}$ & ND & $1.6 \times 10^{4}$ & $1.0 \times 10^{2}$ & ND \\
\hline TC $0.0025 \%$ & $4.7 \times 10^{7}$ & ND & $8.0 \times 10^{4}$ & ND & ND \\
\hline TC $0.005 \%$ & $1.5 \times 10^{8}$ & ND & $1.0 \times 10^{4}$ & $1.4 \times 10^{2}$ & ND \\
\hline TC $0.01 \%$ & $1.6 \times 10^{8}$ & ND & $7.8 \times 10^{3}$ & $1.6 \times 10^{2}$ & ND \\
\hline \multicolumn{6}{|l|}{ Wilted Napier } \\
\hline AC $0.0025 \%$ & $8.1 \times 10^{7}$ & ND & $7.8 \times 10^{3}$ & $1.0 \times 10^{4}$ & $N D$ \\
\hline AC $0.005 \%$ & $1.8 \times 10^{8}$ & ND & $2.1 \times 10^{4}$ & $1.0 \times 10^{3}$ & $N D$ \\
\hline AC $0.01 \%$ & $1.4 \times 10^{8}$ & ND & $1.5 \times 10^{4}$ & $4.9 \times 10^{3}$ & $N D$ \\
\hline TC $0.0025 \%$ & $7.6 \times 10^{8}$ & ND & $6.6 \times 10^{3}$ & $7.8 \times 10^{3}$ & ND \\
\hline TC $0.005 \%$ & $1.0 \times 10^{8}$ & ND & $4.7 \times 10^{3}$ & $5.1 \times 10^{3}$ & ND \\
\hline TC $0.01 \%$ & $7.3 \times 10^{8}$ & ND & $2.6 \times 10^{4}$ & $1.4 \times 10^{4}$ & ND \\
\hline SEM & 1.331 & ND & 2.037 & 57.895 & ND \\
\hline \multicolumn{6}{|l|}{ Grasses means } \\
\hline Guinea grass & $1.9 \times 10^{8}$ & ND & $1.2 \times 10^{4}$ & $1.6 \times 10^{2}$ & ND \\
\hline Napier grass & $1.1 \times 10^{8}$ & ND & $2.1 \times 10^{4}$ & $4.8 \times 10^{3}$ & ND \\
\hline \multicolumn{6}{|l|}{ Moisture adjust } \\
\hline Fresh & $1.6 \times 10^{8}$ & ND & $1.9 \times 10^{4}$ & $1.3 \times 10^{2}$ & ND \\
\hline Wilted & $1.4 \times 10^{8}$ & ND & $1.3 \times 10^{4}$ & $3.7 \times 10^{3}$ & $N D$ \\
\hline \multicolumn{6}{|l|}{ Enzymes } \\
\hline$A C$ & $1.6 \times 10^{8}$ & ND & $1.5 \times 10^{4}$ & $1.4 \times 10^{3}$ & ND \\
\hline TC & $1.4 \times 10^{8}$ & ND & $1.7 \times 10^{4}$ & $3.6 \times 10^{3}$ & ND \\
\hline \multicolumn{6}{|l|}{ Application levels } \\
\hline $0.0025 \%$ & $1.0 \times 10^{8}$ & ND & $1.8 \times 10^{4}$ & $2.3 \times 10^{3}$ & ND \\
\hline $0.005 \%$ & $1.9 \times 10^{8}$ & ND & $1.6 \times 10^{4}$ & $2.7 \times 10^{3}$ & ND \\
\hline $0.01 \%$ & $1.7 \times 10^{8}$ & ND & $1.5 \times 10^{4}$ & $2.5 \times 10^{3}$ & ND \\
\hline \multicolumn{6}{|c|}{ Significance of main effect and interaction } \\
\hline Grasses means (A) & 0.088 & ND & 0.239 & 0.250 & ND \\
\hline Moisture adjust (B) & 0.779 & ND & 0.341 & 0.243 & ND \\
\hline Enzymes (C) & 0.675 & ND & 0.790 & 0.294 & ND \\
\hline Application levels (D) & 0.271 & ND & 0.943 & 0.991 & ND \\
\hline$A \times B \times C \times D$ & 0.939 & ND & 0.660 & 0.878 & ND \\
\hline
\end{tabular}

FM, fresh matter; ND, not detected; AC, Acremonium cellulase (Meiji Seika Pharma Co. Ltd., Tokyo, Japan); TC, Trichoderma cellulase (Meiji Seika Pharma Co. Ltd., Japan); SEM, standard error of the mean.

Values are means of 3 silage samples.

dominated by clostridium, resulting in low quality silage production [28]. In this study, the DM content of fresh Guinea
Table 5. Chemical compositions (\% of DM) of Guinea grass and Napier grass silages at $30 \mathrm{~d}$ after fermentation

\begin{tabular}{|c|c|c|c|c|c|c|}
\hline Treatments & $\mathrm{OM}$ & $\mathrm{CP}$ & $\mathrm{EE}$ & NDF & ADF & ADL \\
\hline \multicolumn{7}{|l|}{ Fresh Guinea } \\
\hline AC $0.0025 \%$ & $90.91^{\mathrm{a}}$ & $6.64^{\mathrm{ef}}$ & $2.85^{\text {abcde }}$ & $75.58^{\mathrm{ab}}$ & $50.29^{\text {cde }}$ & $4.18^{\text {bcdef }}$ \\
\hline AC $0.005 \%$ & $90.54^{\mathrm{a}}$ & $6.26^{f g}$ & $2.78^{\text {abcde }}$ & $74.82^{b}$ & $49.54^{\text {de }}$ & $4.57^{\mathrm{abcd}}$ \\
\hline AC $0.01 \%$ & $90.83^{\mathrm{a}}$ & $6.76^{\mathrm{de}}$ & $2.36^{f g}$ & $71.04^{\text {de }}$ & $46.17^{f}$ & $3.91^{\text {cdefgh }}$ \\
\hline TC $0.0025 \%$ & $90.51^{\mathrm{a}}$ & $5.84^{\mathrm{hij}}$ & $2.72^{\text {abcdefg }}$ & $75.36^{\mathrm{ab}}$ & $51.84^{\mathrm{ab}}$ & $5.22^{\mathrm{a}}$ \\
\hline TC $0.005 \%$ & $90.31^{\mathrm{a}}$ & $6.26^{f g}$ & $2.64^{\text {cdefg }}$ & $75.12^{\mathrm{ab}}$ & $50.97^{\mathrm{bc}}$ & $4.34^{\text {abcde }}$ \\
\hline TC $0.01 \%$ & $90.60^{\mathrm{a}}$ & $5.69^{\mathrm{ijk}}$ & $2.68^{\text {bcdefg }}$ & $76.48^{\mathrm{a}}$ & $52.67^{\mathrm{a}}$ & $4.87^{\mathrm{ab}}$ \\
\hline \multicolumn{7}{|l|}{ Wilted Guinea } \\
\hline AC $0.0025 \%$ & $89.18^{b}$ & $7.33^{\mathrm{bc}}$ & $2.92^{\text {abcd }}$ & $70.69^{e}$ & $49.63^{d e}$ & $2.64^{j}$ \\
\hline AC $0.005 \%$ & $89.16^{b}$ & $7.34^{\mathrm{bc}}$ & $2.97^{\mathrm{abc}}$ & $70.30^{\mathrm{e}}$ & $49.12^{\mathrm{e}}$ & $4.14^{\text {bcdef }}$ \\
\hline AC $0.01 \%$ & $89.26^{b}$ & $6.91^{\mathrm{de}}$ & $3.08^{\mathrm{ab}}$ & $68.46^{f}$ & $47.31^{f}$ & $4.67^{\mathrm{abc}}$ \\
\hline TC $0.0025 \%$ & $89.05^{b}$ & $7.05^{\mathrm{cd}}$ & $2.68^{\text {bcdefg }}$ & $72.22^{\text {cd }}$ & $50.56^{\text {bcd }}$ & $3.99^{\text {bcdeff }}$ \\
\hline TC $0.005 \%$ & $89.01^{b}$ & $7.64^{b}$ & $2.84^{\text {abcde }}$ & $72.52^{c}$ & $50.67^{\text {bcd }}$ & $3.54^{\text {efghij }}$ \\
\hline TC $0.01 \%$ & $89.28^{b}$ & $7.71^{\mathrm{b}}$ & $3.09^{\mathrm{ab}}$ & $72.42^{\text {cd }}$ & $51.42^{\mathrm{abc}}$ & $2.92^{i j}$ \\
\hline \multicolumn{7}{|l|}{ Fresh Napier } \\
\hline AC $0.0025 \%$ & $86.05^{d}$ & $5.85^{\mathrm{hij}}$ & $2.74^{\text {abcdef }}$ & $65.27^{\mathrm{ij}}$ & $41.62^{h}$ & $3.69^{\text {defgh }}$ \\
\hline AC $0.005 \%$ & $87.22^{c}$ & $5.56^{\mathrm{jk}}$ & $2.98^{\mathrm{abc}}$ & $67.64^{\text {fg }}$ & $43.01^{9}$ & $4.31^{\text {bcde }}$ \\
\hline AC $0.01 \%$ & $86.77^{c}$ & $5.97^{\text {ghi }}$ & $3.02^{\mathrm{abc}}$ & $66.57^{\text {ghi }}$ & $42.10^{\text {gh }}$ & $4.68^{\mathrm{abc}}$ \\
\hline TC $0.0025 \%$ & $85.72^{d}$ & $6.14^{\text {gh }}$ & $2.75^{\text {abcdef }}$ & $67.10^{\mathrm{fgh}}$ & $43.03^{9}$ & $2.83^{i j}$ \\
\hline TC $0.005 \%$ & $84.59^{\mathrm{e}}$ & $5.55^{j k}$ & $2.32^{9}$ & $65.24^{i j}$ & $42.01^{\text {gh }}$ & $2.65^{j}$ \\
\hline TC $0.01 \%$ & $85.84^{d}$ & $5.34^{k}$ & $2.46^{\text {efg }}$ & $66.29^{\text {ghi }}$ & $43.17^{9}$ & $2.81^{i j}$ \\
\hline \multicolumn{7}{|l|}{ Wilted Napier } \\
\hline AC $0.0025 \%$ & $85.46^{d}$ & $7.67^{b}$ & $2.90^{\mathrm{abcd}}$ & $64.49^{\mathrm{jk}}$ & $38.82^{j}$ & $3.02^{\mathrm{hij}}$ \\
\hline AC $0.005 \%$ & $85.39^{d}$ & $7.71^{\mathrm{b}}$ & $2.69^{\text {bcdefg }}$ & $63.34^{k l}$ & $37.97^{\mathrm{k}}$ & $3.37^{\text {fohij }}$ \\
\hline AC $0.01 \%$ & $85.89^{d}$ & $8.25^{\mathrm{a}}$ & $2.89^{\text {abcd }}$ & $62.79^{\prime}$ & $36.89^{k}$ & $3.28^{\text {fghij }}$ \\
\hline TC $0.0025 \%$ & $85.98^{d}$ & $7.59^{\mathrm{b}}$ & $2.71^{\text {abcdefg }}$ & $64.32^{j^{k}}$ & $40.05^{i}$ & $2.85^{\mathrm{ij}}$ \\
\hline TC $0.005 \%$ & $85.50^{d}$ & $7.51^{\mathrm{b}}$ & $3.12^{\mathrm{a}}$ & $66.42^{\text {ghi }}$ & $40.26^{i}$ & $3.04^{\mathrm{hij}}$ \\
\hline TC $0.01 \%$ & $85.50^{d}$ & $7.64^{b}$ & $2.53^{\text {defg }}$ & $66.09^{\mathrm{hi}}$ & $40.28^{i}$ & $3.17^{\text {ghij }}$ \\
\hline SEM & 0.265 & 0.148 & 0.141 & 0.551 & 0.489 & 0.322 \\
\hline \multicolumn{7}{|l|}{ Grasses means } \\
\hline Guinea grass & $89.89^{\mathrm{a}}$ & 6.79 & 2.80 & $72.92^{\mathrm{a}}$ & $50.02^{\mathrm{a}}$ & $4.08^{\mathrm{a}}$ \\
\hline Napier grass & $85.83^{b}$ & 6.73 & 2.76 & $65.46^{b}$ & $40.77^{b}$ & $3.31^{b}$ \\
\hline \multicolumn{7}{|l|}{ Moisture adjust } \\
\hline Fresh & $88.32^{\mathrm{a}}$ & $5.99^{b}$ & $2.69^{b}$ & $70.54^{\mathrm{a}}$ & $46.37^{\mathrm{a}}$ & $4.00^{\mathrm{a}}$ \\
\hline Wilted & $87.39^{b}$ & $7.53^{\mathrm{a}}$ & $2.87^{\mathrm{a}}$ & $67.84^{b}$ & $44.41^{b}$ & $3.39^{b}$ \\
\hline \multicolumn{7}{|l|}{ Enzymes } \\
\hline$A C$ & $88.06^{\mathrm{a}}$ & $6.85^{\mathrm{a}}$ & $2.85^{\mathrm{a}}$ & $68.42^{\mathrm{b}}$ & $44.37^{b}$ & $3.87^{\mathrm{a}}$ \\
\hline $\mathrm{TC}$ & $87.66^{b}$ & $6.66^{b}$ & $2.71^{b}$ & $69.97^{\mathrm{a}}$ & $46.41^{\mathrm{a}}$ & $3.52^{b}$ \\
\hline \multicolumn{7}{|l|}{ Application levels } \\
\hline $0.0025 \%$ & $87.86^{\mathrm{a}}$ & 6.77 & 2.78 & $69.38^{\mathrm{a}}$ & $45.73^{\mathrm{a}}$ & 3.55 \\
\hline $0.005 \%$ & $87.72^{b}$ & 6.73 & 2.79 & $69.43^{\mathrm{a}}$ & $45.44^{\mathrm{a}}$ & 3.74 \\
\hline $0.01 \%$ & $87.99^{\mathrm{a}}$ & 6.78 & 2.76 & $68.77^{b}$ & $45.00^{b}$ & 3.79 \\
\hline \multicolumn{7}{|c|}{ Significance of main effect and interaction } \\
\hline Grasses means $(A)$ & $<0.001$ & 0.306 & 0.396 & $<0.001$ & $<0.001$ & $<0.001$ \\
\hline Moisture adjust (B) & $<0.001$ & $<.001$ & 0.001 & $<0.001$ & $<0.001$ & $<0.001$ \\
\hline Enzymes (C) & $<0.001$ & 0.001 & 0.009 & $<0.001$ & $<0.001$ & 0.003 \\
\hline Application levels (D) & 0.059 & 0.666 & 0.881 & 0.013 & 0.005 & 0.207 \\
\hline$A \times B \times C \times D$ & $<0.001$ & $<0.001$ & 0.002 & $<0.001$ & $<0.001$ & $<0.001$ \\
\hline
\end{tabular}

$\mathrm{DM}$, dry matter; $\mathrm{OM}$, organic matter; $\mathrm{CP}$, crude protein; $\mathrm{EE}$, ether extract; $N D F$, neutral detergent fiber; $A D F$, acid detergent fiber; $A D L$, acid detergent lignin; $A C$, Acremonium cellulase (Meiji Seika Pharma Co. Ltd., Tokyo, Japan); TC, Trichoderma cellulase (Meiji Seika Pharma Co. Ltd., Japan); SEM, standard error of the mean.

${ }^{a-1}$ Means within columns with different superscripts differ at $p<0.05$.

Values are means of three silage samples.

grass and Napier grass were lower than $18 \%$, which was not ideal to preserve the forage. When both grasses were wilted 
Table 6. In vitro dry matter digestibility (IVDMD, \% of DM) and in vitro organic matter digestibility (IVOMD, \% of DM) at 6 and $48 \mathrm{~h}$ of incubation of Guinea grass and Napier grass silages at $30 \mathrm{~d}$ after fermentation

\begin{tabular}{|c|c|c|c|c|}
\hline \multirow{2}{*}{ Treatments } & \multicolumn{2}{|c|}{ IVDMD } & \multicolumn{2}{|c|}{ IVOMD } \\
\hline & $6 \mathrm{~h}$ & $48 \mathrm{~h}$ & $6 \mathrm{~h}$ & $48 \mathrm{~h}$ \\
\hline \multicolumn{5}{|l|}{ Fresh Guinea } \\
\hline AC $0.0025 \%$ & $30.02^{\text {abcdef }}$ & $55.62^{\text {fghij }}$ & $36.98^{\mathrm{bcd}}$ & $64.85^{\mathrm{ef}}$ \\
\hline AC $0.005 \%$ & $30.46^{\text {abcdef }}$ & $54.85^{\text {ghij }}$ & $36.01^{\text {cd }}$ & $64.38^{\text {ef }}$ \\
\hline AC $0.01 \%$ & $32.21^{\mathrm{abcd}}$ & $60.76^{\text {cdefgh }}$ & $42.65^{\mathrm{abc}}$ & $67.17^{\text {cdef }}$ \\
\hline TC $0.0025 \%$ & $26.69^{\text {cdef }}$ & $54.05^{i j}$ & $36.29^{\text {cd }}$ & $64.09^{\text {ef }}$ \\
\hline TC $0.005 \%$ & $25.16^{\text {ef }}$ & $54.43^{\mathrm{hij}}$ & $35.81^{\text {cd }}$ & $64.54^{\text {ef }}$ \\
\hline TC $0.01 \%$ & $26.36^{\text {def }}$ & $52.19^{j}$ & $36.06^{\text {cd }}$ & $61.68^{f}$ \\
\hline \multicolumn{5}{|l|}{ Wilted Guinea } \\
\hline AC $0.0025 \%$ & $27.99^{\text {bcdef }}$ & $59.48^{\text {defghi }}$ & $38.17^{\mathrm{bcd}}$ & $68.73^{\text {bcde }}$ \\
\hline AC $0.005 \%$ & $31.14^{\text {abcdef }}$ & $57.38^{\text {efghij }}$ & $40.96^{\mathrm{abcd}}$ & $66.62^{\text {def }}$ \\
\hline AC $0.01 \%$ & $34.59^{\mathrm{ab}}$ & $59.44^{\text {defghi }}$ & $44.23^{a b}$ & $71.89^{\text {abcd }}$ \\
\hline TC $0.0025 \%$ & $24.83^{f}$ & $57.12^{\text {efghij }}$ & $34.91^{d}$ & $66.21^{\text {def }}$ \\
\hline TC $0.005 \%$ & $32.68^{\mathrm{abcd}}$ & $58.51^{\text {defghi }}$ & $39.29^{\text {bcd }}$ & $68.12^{\text {bcde }}$ \\
\hline TC $0.01 \%$ & $29.52^{\text {abcdef }}$ & $59.23^{\text {defghi }}$ & $38.83^{\mathrm{bcd}}$ & $68.23^{\text {bcde }}$ \\
\hline \multicolumn{5}{|l|}{ Fresh Napier } \\
\hline AC $0.0025 \%$ & $29.12^{\text {abcdef }}$ & $57.35^{\text {efghij }}$ & $38.57^{\mathrm{bcd}}$ & $67.31^{\text {def }}$ \\
\hline AC $0.005 \%$ & $33.22^{\mathrm{abcd}}$ & $66.26^{\mathrm{abc}}$ & $42.98^{\mathrm{abc}}$ & $73.32^{a b c}$ \\
\hline AC $0.01 \%$ & $35.60^{\mathrm{a}}$ & $69.68^{\mathrm{a}}$ & $41.33^{\mathrm{abcd}}$ & $75.77^{\mathrm{a}}$ \\
\hline TC $0.0025 \%$ & $33.87^{\mathrm{ab}}$ & $68.12^{\mathrm{ab}}$ & $41.14^{\text {abcd }}$ & $73.74^{\mathrm{ab}}$ \\
\hline TC $0.005 \%$ & $30.19^{\text {abcdef }}$ & $61.45^{\text {cdef }}$ & $38.92^{\mathrm{bcd}}$ & $72.08^{\text {abcd }}$ \\
\hline TC $0.01 \%$ & $31.96^{\text {abcde }}$ & $62.42^{\text {bcde }}$ & $40.61^{\text {abcd }}$ & $73.35^{a b c}$ \\
\hline \multicolumn{5}{|l|}{ Wilted Napier } \\
\hline AC $0.0025 \%$ & $32.47^{\mathrm{abcd}}$ & $62.34^{\text {bcde }}$ & $42.58^{\mathrm{abc}}$ & $71.56^{\text {abcd }}$ \\
\hline AC $0.005 \%$ & $33.53^{\mathrm{abc}}$ & $64.55^{\mathrm{abcd}}$ & $41.62^{\mathrm{abcd}}$ & $73.84^{\mathrm{ab}}$ \\
\hline AC $0.01 \%$ & $35.02^{\mathrm{ab}}$ & $66.69^{\mathrm{abc}}$ & $47.15^{\mathrm{a}}$ & $76.07^{\mathrm{a}}$ \\
\hline TC $0.0025 \%$ & $31.77^{\text {abcdef }}$ & $63.98^{\mathrm{abcd}}$ & $40.71^{\mathrm{abcd}}$ & $73.38^{\mathrm{abc}}$ \\
\hline TC $0.005 \%$ & $34.15^{\mathrm{ab}}$ & $61.13^{\text {cdefg }}$ & $42.76^{\mathrm{abc}}$ & $71.10^{\text {abcd }}$ \\
\hline TC $0.01 \%$ & $32.94^{\mathrm{abcd}}$ & $64.84^{\mathrm{abcd}}$ & $41.65^{\mathrm{abcd}}$ & $74.19^{\mathrm{ab}}$ \\
\hline SEM & 2.392 & 2.184 & 2.499 & 2.165 \\
\hline \multicolumn{5}{|l|}{ Grasses means } \\
\hline Guinea grass & $29.31^{b}$ & $38.35^{b}$ & $56.92^{b}$ & $66.38^{b}$ \\
\hline Napier grass & $32.82^{\mathrm{a}}$ & $41.67^{\mathrm{a}}$ & $64.07^{\mathrm{a}}$ & $72.97^{\mathrm{a}}$ \\
\hline \multicolumn{5}{|l|}{ Moisture adjust } \\
\hline Fresh & 30.41 & 59.76 & $38.95^{b}$ & $68.52^{b}$ \\
\hline Wilted & 31.72 & 61.22 & $41.07^{\mathrm{a}}$ & $70.83^{\mathrm{a}}$ \\
\hline \multicolumn{5}{|l|}{ Enzymes } \\
\hline$A C$ & $32.11^{\mathrm{a}}$ & 61.20 & $41.10^{\mathrm{a}}$ & 70.12 \\
\hline TC & $30.01^{b}$ & 59.79 & $38.92^{b}$ & 69.23 \\
\hline \multicolumn{5}{|l|}{ Application levels } \\
\hline $0.0025 \%$ & $29.60^{b}$ & $59.76^{b}$ & $38.67^{b}$ & $68.73^{b}$ \\
\hline $0.005 \%$ & $31.32^{\mathrm{ab}}$ & $59.82^{b}$ & $39.79^{a b}$ & $69.25^{\mathrm{ab}}$ \\
\hline $0.01 \%$ & $32.27^{\mathrm{a}}$ & $61.90^{\mathrm{a}}$ & $41.56^{\mathrm{a}}$ & $71.04^{\mathrm{a}}$ \\
\hline \multicolumn{5}{|c|}{ Significance of main effect and interaction } \\
\hline Grasses means (A) & $<0.001$ & $<0.001$ & $<0.001$ & $<0.001$ \\
\hline Moisture adjust (B) & 0.127 & 0.065 & 0.020 & 0.004 \\
\hline Enzymes (C) & 0.016 & 0.074 & 0.017 & 0.246 \\
\hline Application levels (D) & 0.040 & 0.044 & 0.034 & 0.044 \\
\hline$A \times B \times C \times D$ & 0.445 & $<0.001$ & 0.787 & 0.264 \\
\hline
\end{tabular}

AC, Acremonium cellulase (Meiji Seika Pharma Co. Ltd., Tokyo, Japan); TC, Trichoderma cellulase (Meiji Seika Pharma Co. Ltd., Japan); SEM, standard error of the mean.

ath Means within columns with different superscripts differ at $p<0.05$.

Values are means of three silage samples. for $6 \mathrm{~h}$ in the field, DM contents of Guinea grass and Napier grass were higher than $21 \%$, much more suitable for ensiling. The decreasing of moisture during the wilting process under this experiment is negligible and less than those of the optimal DM content ranges. In addition, the wilted both grasses as shown Table 2 had a higher $\mathrm{CP}$ and lower ADF compared with that of fresh forage. However, we cannot fully explain the mechanism of these effects, this may be influenced by the climatic conditions such as low temperature and high humidity during the wilting process. Future study is necessary to study the effect of climatic condition on the chemical composition of forage during wilting process.

The CP, NDF, and ADF of Guinea grass and Napier grass used in this study were similar to our previous work which reported that Guinea grass and Napier grass harvested at $60 \mathrm{~d}$ of regrowth were lower in CP contents, but high in fiber [16]. The WSC in the ensiling material also plays an important role in silage fermentation and it is the main energy source for LAB growth to produce lactic acid [29,30]. Wilkinson [27] suggested that WSC needs to be greater than $10 \%$ of DM for good fermentation. However, the WSC contents of fresh forages in this present study were less than $2.7 \%$ of DM (Table 2). Although a lower WSC content was found in Guinea grass compared to Napier grass, it would be difficult to make good quality silage from both grasses [5].

At $30 \mathrm{~d}$ of fermentation, Guinea grass silages were poor quality. As shown in Tables 1, 2, both fresh and wilted Guinea grasses had low LAB counts and WSC content which were not sufficient to produce more lactic acid for good fermentation. Thus, the $\mathrm{pH}$ of Guinea grass silages did not decrease below 4.2, which cannot inhibit the growth of harmful bacteria, especially clostridia [13]. As a result, a harmful fermentation could occur with clostridia using WSC, amino acid, and other organic acids to produce butyric acid and ammonia nitrogen.

Some protein of forages breaks down to simpler or nonprotein nitrogen during wilting process, result in decreased true protein solubility [31]. Nevertheless, this is consistent with previous studies [32] that total protein content of intact asparagus spear and excised tip sections increased approximately $10 \%$ after 6 to $12 \mathrm{~h}$ of harvested. Also, our results indicated that wilted forages silages were lower $(p<0.01)$ lactic acid content than fresh forage silages. Consistently, our previous study [16] evidenced a lower lactic acid concentration in silages prepared from wilted forages compared to fresh forages. The significantly higher DM in wilted forages silages may attribute to decrease LAB growth and activity during silage fermentation.

When grass silages treated with AC $0.01 \%$, their fermentation quality were more improved compared to other treatments. Our findings are in agreement with [9,33-35]. that the addition of cellulase enzyme resulted in a decrease of $\mathrm{pH}$ and increased lactic acid content in sorghum straw, oil palm frond and mixed hulless-barley straw with corn silages. This could be attributed 
to the cellulase enzyme degrading plant cellulose so increasing the WSC, an essential substrate for LAB growth and more lactic acid production. As a result, the $\mathrm{pH}$ of silages decrease rapidly inhibiting the growth of clostridia and preventing proteolysis in the silo $[33,36]$. Some studies $[15,29,34,37]$ reported that addition of cellulase resulted in a decrease in fiber content of mixed silages of hulless-barley straw and corn, wheat straw, Leymus chinensis, and Napier grass silage. On the other hand, cellulase addition had no effect on NDF and ADF content of barley and vetch silage [38]. In the present study, the AC improved silage fermentation more than TC, and the AC $0.01 \%$ cellulase effectively increased CP content and reduced NDF and ADF contents of silages. Perhaps the type, composition and enzyme activity of cellulase may affect cellulose degradation and silage fermentation. Generally, cellulases catalyze the hydrolysis of cellulose, which are mainly three types: endoglucanases, cellobiohydrolases and $\beta$-glucosidases, and it is used for any occurring mixture or complex of various such enzymes, that act serially or synergistically to decompose cellulosic material. Acremonium cellulolyticus-producing cellulases contain a strong glucanase and pectinase, and cellulase produced by Trichoderma viride contains mainly xylanase and glucanase. Therefore, Acremonium cellulolyticus-producing cellulase is said to be more potent than Trichoderma virideproducing cellulase. This indicated that $\mathrm{AC}$ is more effective for improvement of silage fermentation than TC, and its addition concentration is necessary to be $0.01 \%$ on a FM basis.

The digestibility of roughage also plays an important role in animal production $[10,29]$. The IVDMD and IVOMD depend on physical characteristics of forage, especially the fiber content, low NDF and ADF contents resulted in a rapid increase in digestibility of DM and OM [29,39]. In the present study, Napier grass silages had higher IVDMD and IVOMD when compared to Guinea grass. This could be attributed to the differences in chemical composition of Guinea grass and Napier grass. Guinea grass had higher fiber content than Napier grass as shown in Table 2. Furthermore, the cellulase enzyme addition, especially AC $0.01 \%$ treated silages had greater IVDMD and IVOMD values than other enzyme application levels in both 6 and $48 \mathrm{~h}$ of incubation (Table 6). This study is consistent with previous studies reported by $[10,40]$ that the addition of an exogenous cellulase enzyme could increase IVDMD of forage crops, but inconsistent with [29] that cellulase addition did not increase the IVDMD of Leymus chinensis after $48 \mathrm{~h}$ of incubation. The greater effect of AC $0.01 \%$ treatment in the present study due to the composition of enzymes. The AC used in this study has both glucanase and pectinase; the synergistic effects of these enzymes probably increase silage digestibility. The CMCase activity is also higher in $\mathrm{AC}(7,350$ $\mathrm{U} / \mathrm{g})$ than TC $(2,720 \mathrm{U} / \mathrm{g})$.

These results confirmed that Napier grass has more suitable ensiling characteristics than Guinea grass, and the wilted silage can decrease the feed nutrient loss and improve the in vitro digestibility. The addition of AC $0.01 \%$ cellulase $(73.5 \mathrm{U} / \mathrm{g}$ of FM) was effective in improving the silage fermentation and promoting the in vitro degradation of tropical grass.

\section{CONCLUSION}

Silage fermentation and in vitro digestibility of Guinea grass and Napier grass prepared with cellulase enzyme at different addition concentration were studied. The AC has the potential to improve the fermentation quality, chemical composition and in vitro degradation of Guinea grass and Napier grass, but the TC did not affect the tropical silage fermentation. The AC addition at $0.01 \%(73.5 \mathrm{U} / \mathrm{g})$ of FM was the most promising for improving silage fermentation containing low WSC.

\section{CONFLICT OF INTEREST}

We certify that there is no conflict of interest with any financial organization regarding the material discussed in the manuscript.

\section{ACKNOWLEDGMENTS}

This work was supported by the Project "The Establishment of the Sustainable and Independent Farm Household Economy in the Rural Areas of Indo-China”, Japan International Research Center for Agricultural Sciences (JIRCAS), Japan. Acknowledgments are extended to Meiji Seika Pharma Company, Ltd., Tokyo, Japan for providing the commercial cellulase enzymes.

\section{REFERENCES}

1. Phitsuwan P, Charupongrat S, Klednark R, Ratanakhanokchai K. Structural features and enzymatic digestibility of Napier grass fibre treated with aqueous ammonia. J Ind Eng Chem 2015;32:360-364.

2. Reddy KO, Maheswari CU, Shukla M, Rajulu AV. Chemical composition and structural characterization of Napier grass fibers. Mater Lett 2012;67:35-38.

3. Hare M, Tatsapong P, Phengphet S. Herbage yield and quality of Brachiaria cultivars, Paspalum atratum and Panicum maximum in north-east Thailand. Trop Grassl 2009;43:65-72.

4. Kiyothong K. Manual for planting Napier pakchong 1. Nakhonrajasrima, Thailand: Department of Livestock Development, Thailand; 2014.

5. Pholsen S, Khota W, Pang H, Higgs D, Cai Y. Characterization and application of lactic acid bacteria for tropical silage preparation. Anim Sci J 2016;87:1202-1211.

6. Zhu Y, Nishino N, Xusheng G. Chemical changes during ensilage and in sacco degradation of two tropical grasses: Rhodesgrass and guineagrass treated with cell wall-degrading enzymes. 
Asian-Australas J Anim Sci 2011;24:214-221.

7. Yitbarek M, Tamir B. Silage additives: review. Open J Appl Sci 2014;4:258-274.

8. Takekhalaf M, Torbatinejad NM, Zerehdaran S, Toghdory AH. Effect of treated alfalfa silage with lactobacillus plant arum and sugar beet pulp molasses on performance of Holstein dairy cows. J Appl Environ Biol Sci 2015;5:254-259.

9. Xing L, Chen LJ, Han LJ. The effect of an inoculant and enzymes on fermentation and nutritive value of sorghum straw silages. Bioresour Technol 2009;100:488-491.

10. Li M, Zi X, Zhou H, Hou G, Cai Y. Effects of sucrose, glucose, molasses and cellulase on fermentation quality and in vitro gas production of king grass silage. Anim Feed Sci Technol 2014;197:206-212.

11. Eun J-S, Beauchemin KA. Relationship between enzymic activities and in vitro degradation of alfalfa hay and corn silage. Anim Feed Sci Technol 2008;145:53-67.

12. Ridla M, Uchida S. Effect of combined treatment of lactic acid bacteria and cell wall degrading enzymes on fermentation and composition of rhodesgrass (Chloris gayana Kunth.) silage. Asian-Australas J Anim Sci 1998;11:522-529.

13. McDonald P, Henderson A, Heron S. The biochemistry of silage. Marlow, UK: Chalcombe Publications; 1991.

14. Li X, Yang H, Roy B, et al. Enhanced cellulase production of the Trichoderma viride mutated by microwave and ultraviolet. Microbiol Res 2010;165:190-198.

15. Desta ST, Yuan XJ, Li J, Shao T. Ensiling characteristics, structural and nonstructural carbohydrate composition and enzymatic digestibility of Napier grass ensiled with additives. Bioresour Technol 2016;221:447-454.

16. Khota W, Pholsen S, Higgs D, Cai Y. Natural lactic acid bacteria population of tropical grasses and their fermentation factor analysis of silage prepared with cellulase and inoculant. J Dairy Sci 2016;99:9768-9781.

17. Yoottasanong C, Pholsen S, Higgs DEB. Dry matter yields and forage quality of grass alone and grass plus legume mixture in relation to cattle manure rates and production methods. Pakistan J Biol Sci 2015;18:324-332.

18. Cai Y, Kumai S, Ogawa M, Benno Y, Nakase T. Characterization and identification of Pediococcus species isolated from forage crops and their application for silage preparation. Appl Environ Microbiol 1999;65:2901-2906.

19. Kozaki M, Uchimura T, Okada S. Experimental manual for lactic acid bacteria. Tokyo, Japan: Asakurasyoten; 1992. p. 29-72.

20. Cai Y. Analysis method for silage. In: Japanese Society of Grassland Science, editor. Field and Laboratory methods for grassland science. Tokyo, Japan: Tosho Printing Co. Ltd.; 2004.

21. Fawcett JK, Scott JE. A rapid and precise method for the determination of urea. J Clin Pathol 1960;13:156-159.

22. AOAC. Official methods of analysis. 15th edn. Arlington, VA, USA: Association of Official Analytical Chemists; 1990.
23. Van Soest PJ, Robertson JB, Lewis BA. Methods for dietary fiber, neutral detergent fiber, and nonstarch polysaccharides in relation to animal nutrition. J Dairy Sci 1991;74:3583-3597.

24. European Commission. "European Union Strategy for the Protection and Welfare of Animals 2012-2015", Communication from the Commission to the European Parliament. The Council and the European Economic and Social Committee; 2002. COM 6 final/2, 15 Feb, 2012.

25. Makkar HP, Blümmel M, Becker K. Formation of complexes between polyvinyl pyrrolidones or polyethylene glycols and tannins, and their implication in gas production and true digestibility in in vitro techniques. Br J Nutr 1995;73:897-913.

26. Steel RGD, Torrie JH. Principles and procedures of statistics: a biometrical approach. 2nd ed. New York, NY, USA: McGrawHill Book Co. Inc.; 1980.

27. Wilkinson M. Silage UK. Great Britain: Cambrian printers; 1990.

28. Nkosi BD, Meeske R, Langa T, Motiang MD, Mutavhatsindi TF. The influence of ensiling potato hash waste with enzyme/ bacterial inoculant mixtures on the fermentation characteristics, aerobic stability and nutrient digestion of the resultant silages by rams. Small Rumin Res 2015;127:28-35.

29. Zhang Q, Yu Z, Yang H, Na RS. The effects of stage of growth and additives with or without cellulase on fermentation and in vitro degradation characteristics of Leymus chinensis silage. Grass Forage Sci 2016;71:595-606.

30. Cai Y, Benno Y, Ogawa M, Kumai S. Effect of applying lactic acid bacteria isolated from forage crops on fermentation characteristics and aerobic deterioration of silage. J Dairy Sci 1999; 82:520-526.

31. Rotz CA, Muck RE. Changes in forage quality during harvest and storage. In: Fahey GC, Collins M, Mertens DR, Moser LE, editors. Forage quality, evaluation, and utilization. Madison, USA: American Society of Agronomy, Crop Science Society of America, Soil Science Society of America; 1994. pp. 828-68.

32. King GA, Woollard DC, Irving DE, Borst WM. Physiological changes in asparagus spear tips after harvest. Physiol Plant 1990;80:393-400.

33. Oladosu Y, Rafii MY, Abdullah N, et al. Fermentation quality and additives: a case of rice straw silage. Biomed Res Int 2016; 2016:1-14.

34. Guo G, Yuan X, Li L, Wen A, Shao T. Effects of fibrolytic enzymes, molasses and lactic acid bacteria on fermentation quality of mixed silage of corn and hulless-barely straw in the Tibetan Plateau. Grassl Sci 2014;60:240-6.

35. Ebrahimi M, Rajion MA, YongMeng G, et al. The effects of adding lactic acid bacteria and cellulase in oil palm (Elais guineensis Jacq.) frond silages on fermentation quality, chemical composition and in vitro digestibility. Ital J Anim Sci 2014;13: 557-62.

36. Nkosi BD, Vadlani PV, Brijwani K, Nanjunda A, Meeske R. Effects of bacterial inoculants and an enzyme on the fermen- 
tation quality and aerobic stability of ensiled whole-crop sweet sorghum. S Afr J Anim Sci 2012;42:232-40.

37. Ni K, Wang Y, Pang H, Cai Y. Effect of cellulase and lactic acid bacteria on fermentation quality and chemical composition of wheat straw silage. Am J Plant Sci 2014;5:1877-84.

38. Kung L, Carmean B, Tung R. Microbial inoculation or cellulase enzyme treatment of barley and vetch silage harvested at three maturities. J Dairy Sci 1990;73:1304-11.
39. Huhtanen P, Rinne M, Nousiainen J. Evaluation of the factors affecting silage intake of dairy cows: a revision of the relative silage dry-matter intake index. Animal 2007;1:758-70.

40. Moharrery A, Hvelplund T, Weisbjerg MR. Effect of forage type, harvesting time and exogenous enzyme application on degradation characteristics measured using in vitro technique. Anim Feed Sci Technol 2009;153:178-92. 